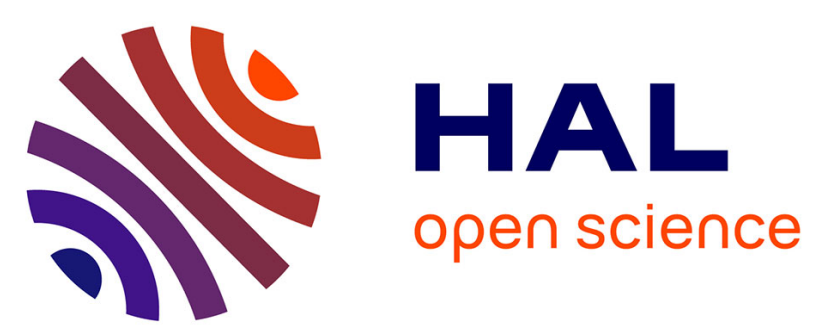

\title{
Nonlinear spectrum broadening cancellation by sinusoidal phase modulation
}

Frederic Audo, Sonia Boscolo, Julien Fatome, Bertrand Kibler, Christophe Finot

\section{- To cite this version:}

Frederic Audo, Sonia Boscolo, Julien Fatome, Bertrand Kibler, Christophe Finot. Nonlinear spectrum broadening cancellation by sinusoidal phase modulation. Optics Letters, 2017, 42 (12), pp.2902-2905. 10.1364/OL.42.002902 . hal-01537838

\section{HAL Id: hal-01537838 \\ https://hal.science/hal-01537838}

Submitted on 13 Jun 2017

HAL is a multi-disciplinary open access archive for the deposit and dissemination of scientific research documents, whether they are published or not. The documents may come from teaching and research institutions in France or abroad, or from public or private research centers.
L'archive ouverte pluridisciplinaire HAL, est destinée au dépôt et à la diffusion de documents scientifiques de niveau recherche, publiés ou non, émanant des établissements d'enseignement et de recherche français ou étrangers, des laboratoires publics ou privés. 


\title{
Nonlinear spectrum broadening cancellation by sinusoidal phase modulation
}

\author{
Frederic Audo, ${ }^{1}$ Sonia Boscolo, ${ }^{2}$ JULien Fatome, ${ }^{1}$ Bertrand Kibler, ${ }^{1}$ AND \\ ChRISTOPHE FINOT ${ }^{1, *}$
}

${ }^{1}$ Laboratoire Interdisciplinaire Carnot de Bourgogne, UMR 6303 CNRS - Université de Bourgogne-Franche-Comté, 9 avenue Alain Savary, BP 47870, 21078 Dijon Cedex, France

${ }^{2}$ Aston Institute of Photonic Technologies, School of Engineering and Applied Science, Aston University, Birmingham B4 7ET, United Kingdom *Corresponding author: christophe.finot@u-bourgogne.fr

Received XX Month XXXX; revised XX Month, XXXX; accepted XX Month XXXX; posted XX Month XXXX (Doc. ID XXXXX); published XX Month XXXX

\begin{abstract}
We propose and experimentally demonstrate a new approach to dramatically reduce the spectral broadening induced by self-phase modulation occurring in a Kerr medium. By using a temporal sinusoidal phase modulation, we efficiently cancel to a large extend the chirp induced by the nonlinear effect. Experimental validation carried out in a passive or amplifying fiber confirm the interest of the technic for the mitigation of spectral expansion of long pulses. (C) 2017 Optical Society of America
\end{abstract}

OCIS codes: (060.2310) Fiber optics amplifiers and oscillators; (060.4370) Nonlinear optics, fibers; (060.5060) Phase modulation.

Whenever an optical signal experiences a temporal modulation of its phase, its spectrum is changed [1]. Such a phase modulation can be externally applied to a signal by an electro-optic modulator for example. But it can also be the result of nonlinear optical effects like the self-phase modulation (SPM) of the signal pulse arising from propagation in a Kerr medium such as an optical fiber [2, 3]. SPM usually causes spectral broadening of a short and intense optical pulse that is accompanied by an oscillatory structure covering the entire frequency range. This may highly impair the suitability of the resulting signal for applications requiring a high spectral resolution or chirp-free pulses. The level of SPM is therefore a limiting factor in fiber amplification, which has to be controlled carefully [4]. Various strategies can be deployed to this end. The most basic one consist in spectrally filtering the broadened spectrum. However, this approach is power inefficient and may induce large distortions of the pulse temporal profile. By stretching the pulses in time before amplification, chirped pulse amplification (CPA) has been extremely successful in mitigating undesirable SPM [5]. By subsequent cancellation of the linearly time varying part of the SPM-induced chirp, the pulse may also be temporally recompressed [6]. However, while the temporal intensity profile can be shortened to approach the Fourier transform limit, no narrowing of the expanded spectrum is attained with this method. Recent approaches to improve the performance of short-pulse fiber amplifiers include the exploitation of the peculiar dynamics of parabolic self-similar pulses [7], the use of pre-shaped input pulses [8], and the compensation of nonlinear phase shifts with thirdorder dispersion [9]. However, all of these techniques do not preserve the pulse temporal duration.

In this contribution we introduce a new method to mitigate nonlinear impairments of optical pulses propagating through fiberoptic systems. The proposed approach is based on the simple observation that the chirp induced by SPM on a pulse with a standard temporal intensity profile, such as a Gaussian or sech ${ }^{2}$ shape, features a close-to-sinusoidal variation in the central pulse region. Hence, by applying to the pulse a sinusoidal modulation of the temporal phase with appropriate amplitude and frequency, we are able to reduce the nonlinear spectrum broadening to a large degree, and to significantly enhance the spectral quality of the pulse while its temporal duration remains unaffected.

In order to illustrate our idea and provide some simple analytical design rules, we consider an initial chirp-free pulse with a Gaussian temporal intensity profile: $G(t)=\exp \left(-t^{2} / T_{0}^{2}\right)$, where $T_{0}$ is the $1 / e$ intensity half-duration, propagating in a purely nonlinear fiber of length $L o$. As a result of SPM, the pulse acquires a nonlinear phase shift $\varphi_{N L}(t)$, hence, an instantaneous frequency shift $\delta \omega_{N L}(t)$ that is proportional to the opposite of the temporal gradient of the intensity profile $\delta \omega_{N L}(t)=-d \varphi_{N L} / d t=-2 B t G(t) / T 0^{2}$. Here, $B=\gamma P_{0} L$ is the maximum phase shift that occurs at the pulse center located at $t=0$ and is widely known as the $B$-integral [6], $P_{0}$ is the peak pulse power, $\gamma$ is the Kerr-nonlinearity coefficient of the fiber, $L=\left(1-\exp \left(-\alpha L_{0}\right)\right) / \alpha$ is the effective propagated length, and $\alpha>0$ $(<0)$ is the fiber loss (gain) coefficient [3]. Figure 1 shows the pulse characterizations at the fiber output for a $B$-integral accumulated in the fiber of $B=5 \mathrm{rad}$. The induced chirp $\delta \omega_{\mathrm{NL}}$ has a non-monotonic temporal variation across the pulse with two extrema around the pulse center (panel (a), red curve). The time separation between these extrema and the corresponding frequency excursion can be computed by $\Delta \tau=\sqrt{2} T_{0}$ and $\Delta \omega=B \sqrt{2} \exp (-1 / 2) / T_{0}$, 
respectively [3]. The pulse spectrum has been significantly broadened over its initial width by the SPM-generated frequency components and has developed a multi-peak structure (panel (b)).

The observed time dependence of $\delta \omega_{N L}(t)$ suggests that this chirp can be offset by applying to the pulse a corrective sinusoidal temporal phase modulation, $\varphi_{c}(t)=A \cos \left(\omega_{c} t+\phi_{0}\right)$, producing the chirp $\delta \omega_{c}=A \omega_{c} \sin \left(\omega_{c} t+\phi_{0}\right)$. Inspired by a previous numerical work relating to the optimization of the SPM-driven spectral compression process [10], we choose the angular frequency, phase shift and amplitude of the modulation as $\omega_{c}=\pi / \Delta \tau=\pi /\left(\sqrt{2} T_{0}\right), \quad \phi_{0}=\pi$ and $A=\Delta \omega / \omega c=2 B \exp (-1 / 2) / \pi$, respectively. With this parameter choice, the external phase modulation indeed compensates the nonlinear phase accumulated by the Gaussian pulse to a large degree: the total chirp, $\delta \omega_{c}+\delta \omega_{N L}$, is now cancelled almost completely over the central region of the pulse (Fig. 1(a), blue curve). This entails a significantly narrower spectrum that is now close to its original width (Fig. 1(b1)). Small pedestals are still present in the spectrum, but their intensity level is below $20 \mathrm{~dB}$. A measure of how close a pulse is to its Fourier transform limit is provided by the spectral Strehl ratio, defined as the ratio of the maximum spectral brilliance of the actual pulse to the brilliance obtained assuming a transform-limited pulse. While after nonlinear propagation in the fiber, the maximum relative brilliance of the pulse spectrum has dropped to 0.25 , the application of the external phase modulation re-establishes a Strehl ratio of 0.7 .
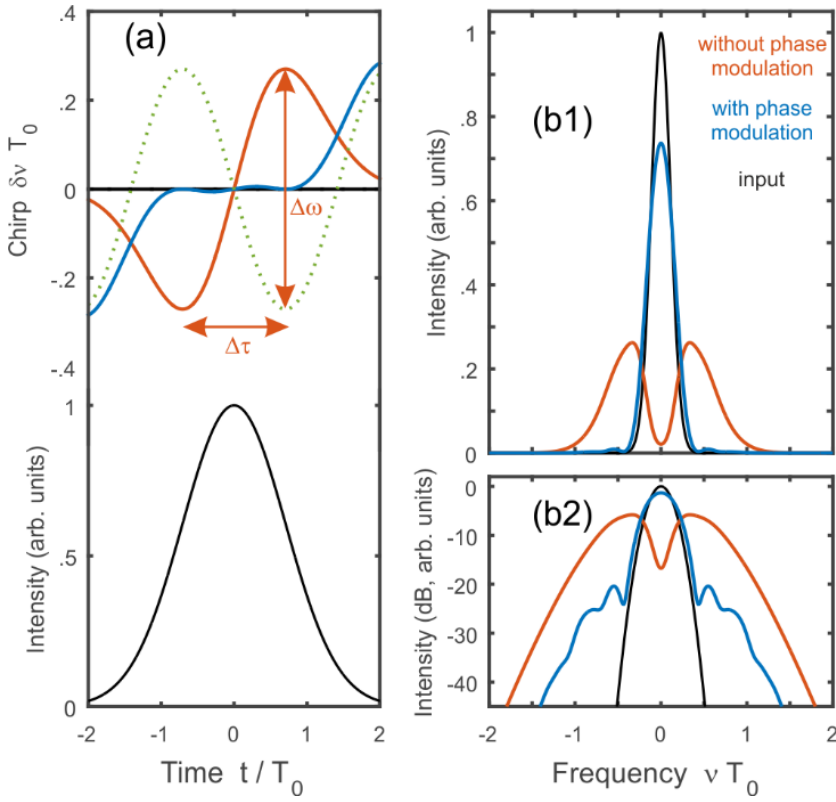

Fig. 1. Numerically computed impact of phase correction on a Gaussian pulse after undergoing SPM: (a) temporal intensity and chirp profiles, and (b) spectral intensity profile on linear and logarithmic scales at the entrance (black) and the exit of the fiber before (red) and after (blue) phase compensation. The $B$-integral accumulated by the pulse is $B=5$ rad. The corrective chirp $\delta v_{c}$ is plotted with a green dotted line.

We have also assessed the ability of our approach to counteract larger amounts of SPM. The results are summarized in Fig. 2, highlighting the excellent mitigation of SPM provided by the corrective phase modulation for values of the accumulated $B$ integral as high as $10 \mathrm{rad}$ : the full-width at half maximum (fwhm) of the spectrum remains constant and no spectral ripple develops.
Further, the corrective approach enables a reduction of the rootmean-square (rms) spectrum broadening by a factor close to 4 relative to the case when no phase correction is used, and the Strehl ratio of the pulse remains well above 0.5 .

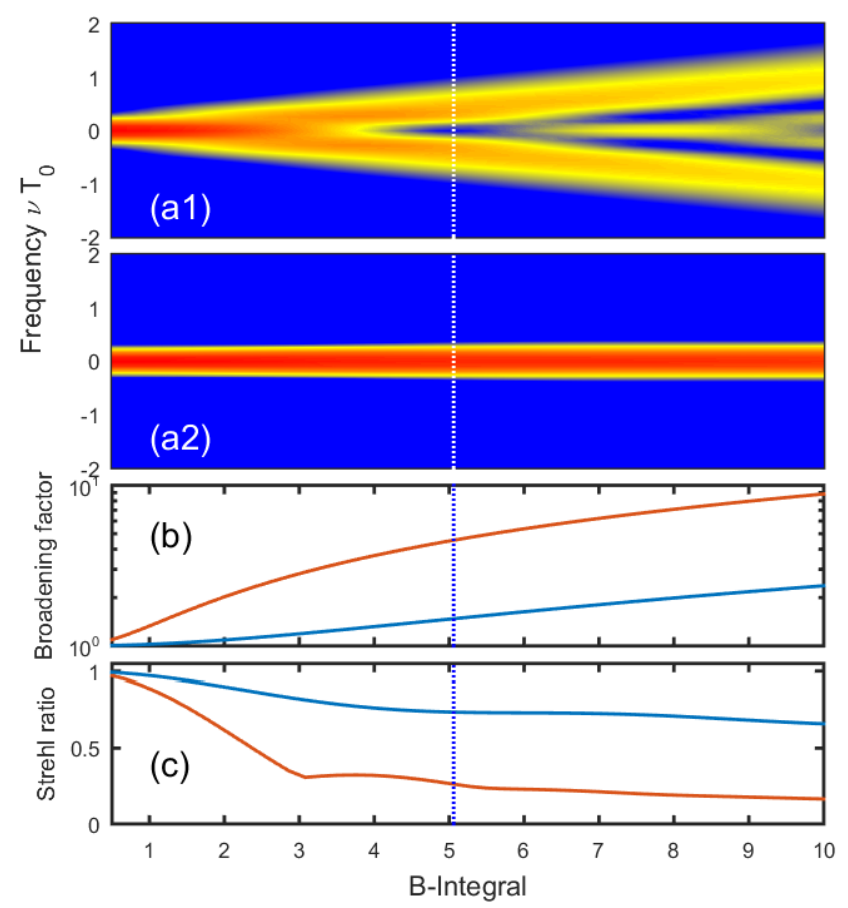

Fig. 2. (a) Numerically computed evolution of the spectrum (logarithmic scale, $17 \mathrm{~dB}$ dynamics) of an initial Gaussian pulse after undergoing SPM with the accumulated $B$-integral, before (subplot 1 ) and after (subplot 2) phase correction. (b)-(c) Evolutions of the rms broadening factorand the Strehl ratio of the pulse at the exit of the fiber. The results obtained without and with phase correction are plotted with red and blue lines, respectively. The vertical dotted line indicates the $B$-integral value used in Fig. 1.

For our proof-of-concept, we used the all-fibered experimental setup depicted in Fig. 3, which relies exclusively on commercially available components from the telecom industry. An external cavity laser at $1550 \mathrm{~nm}$ delivers a continuous wave that is intensity modulated by a lithium-niobate modulator with a bandwidth of $20 \mathrm{GHz}$. The electrical signal that drives the modulator is produced by electrical filtering with a $5.4-\mathrm{GHz}$ low-pass filter of an on-off keyed signal from a 10-GHz pattern generator. A 100-bit sequence with 1 one followed by 99 zeros is chosen to reduce the pulse repetition rate to $100 \mathrm{MHz}$. In our approach in which we assume a purely nonlinear propagation not liable to dispersion-induced distortions, the disposition of the nonlinear element and the external phase modulator does not impact the overall nonlinearitymitigation process. Therefore, the sinusoidal phase modulation can be imparted as a pre-propagation compensation rather than a postpropagation correction. It is noteworthy that in this configuration, the process can be reinterpreted as spectral focusing with the nonlinear phase accumulated by the pulse in propagation compensating an initial and opposite phase modulation [11, 12]. Besides, placing the phase modulator before the fiber has the practical advantages of reducing the threshold of the deleterious Brillouin backscattering and avoiding damages to the optoelectronic component due to excessive optical power. Our 
phase modulator is driven by a $\omega_{c}=4 \mathrm{GHz}$ electrical clock synchronized with the first electrical signal, and imprints a sinusoidal phase with the appropriate phase offset $\phi_{0}$ and amplitude $A$ on the optical wave.
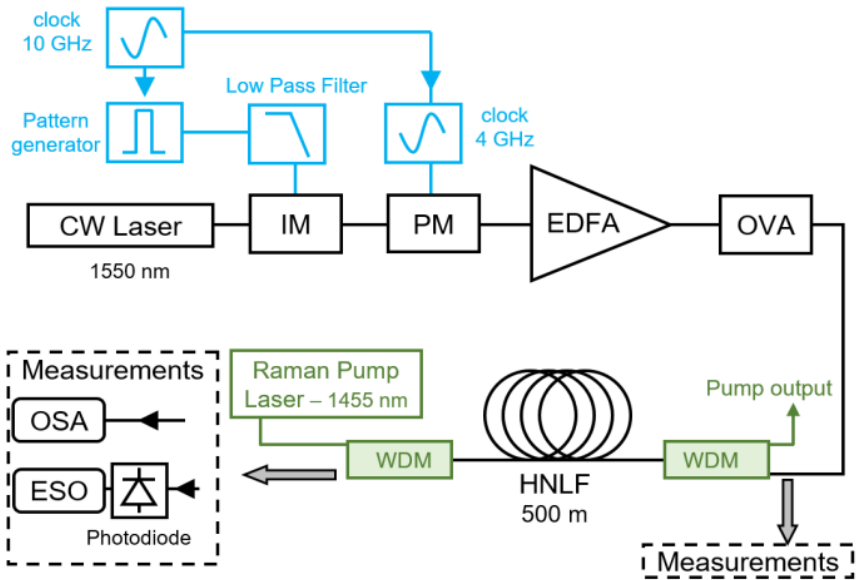

Fig. 3. Experimental setup. CW : continuous wave ; IM : intensity modulator; PM : phase modulator ; EDFA : erbium doped fiber amplifier ; OVA : optical variable attenuator; HNLF : highly nonlinear fiber ; OSA : optical spectrum analyzer; ESO : electrical sampling oscilloscope; WDM : wavelength multiplexer

The resulting signal is amplified by an erbium-doped fiber amplifier and propagated through a 500-m long section of HNLF with the nonlinear coefficient $\gamma=10 \mathrm{~W}^{-1} \cdot \mathrm{km}^{-1}$ and linear attenuation $\alpha=0.7 \mathrm{~dB} / \mathrm{km}$. The group-velocity dispersion (GVD) of the fiber is normal $(D=-1 \mathrm{ps} / \mathrm{km} / \mathrm{nm})$ to inhibit any signal degradation that may be caused by modulation instability. Two wavelength multiplexers and a 1455-nm quasi-continuous laser with a Wattlevel power can turn the passive fiber into a Raman amplifier. We favoured a counter-propagating pump configuration to avoid the emergence of extreme fluctuations [13]. The optical signal is characterized in the time and frequency domains with a $40-\mathrm{GHz}$ electrical sampling oscilloscope and a high-resolution (100 MHz) optical spectrum analyzer (APEX 2443B). The value of the accumulated $B$-integral is varied by control of the peak pulse power at the fiber input with an optical variable attenuator. We note that the finite extinction ratio of the intensity modulator $(23 \mathrm{~dB})$ may cause a small residual coherent background. For clarity of the figures, we have numerically removed its signature, i.e. a coherent spectral line at $1550 \mathrm{~nm}$, from the spectral measurements.

The experimental results obtained for $B=5 \mathrm{rad}$ are plotted in Fig. 4. The initial pulses are Gaussian-like in shape and have fwhm duration of 120 ps. The temporal gradient of the pulse intensity numerically extracted from the experimental data (panel (a2)) shows that the SPM-induced chirp variation can be well approximated by a sinusoidal function (green curve) albeit featuring a small asymmetry. The pulse intensity profile at the fiber output is fully identical to the input, confirming our assumption that with the fiber and temporal pulse durations being used, the propagation regime is purely nonlinear and is not affected by chromatic dispersion. The initial spectrum of the pulse has significantly broadened after propagation in the HNLF, with a spectral width that has more than doubled (see Fig. 4(b1)). Note that the asymmetry in the intensity temporal gradient has been recast into a marked asymmetry of the output spectrum. When the external sinusoidal phase modulation with the appropriate frequency, amplitude and phase is switched on, the spectrum is mostly unperturbed by nonlinear propagation. Some small degradation is visible in the output spectrum when plotted on a logarithmic scale (Fig. 4(b2)). It stems from imperfect phase compensation due to the asymmetry of the input pulse profile and its deviation from the Gaussian shape used in the theoretical model.

Figure 5 summarizes the results obtained with and without phase pre-compensation for different pulse powers injected into the fiber up to $B=5 \mathrm{rad}$. While in the absence of phase correction we observe progressive expansion of the output spectrum from the fiber with increasing values of the accumulated $B$-integral, accompanied by the development of ripple and asymmetry, the use of a corrective phase modulation maintains an output spectrum that is only marginally affected. The benefits afforded by the phase correction method are also apparent in Figs. 5(b)-(c) when comparing the evolution of the rms spectral width and the maximum spectral brilliance of the pulse with the accumulated $B$ integral between the two configurations.
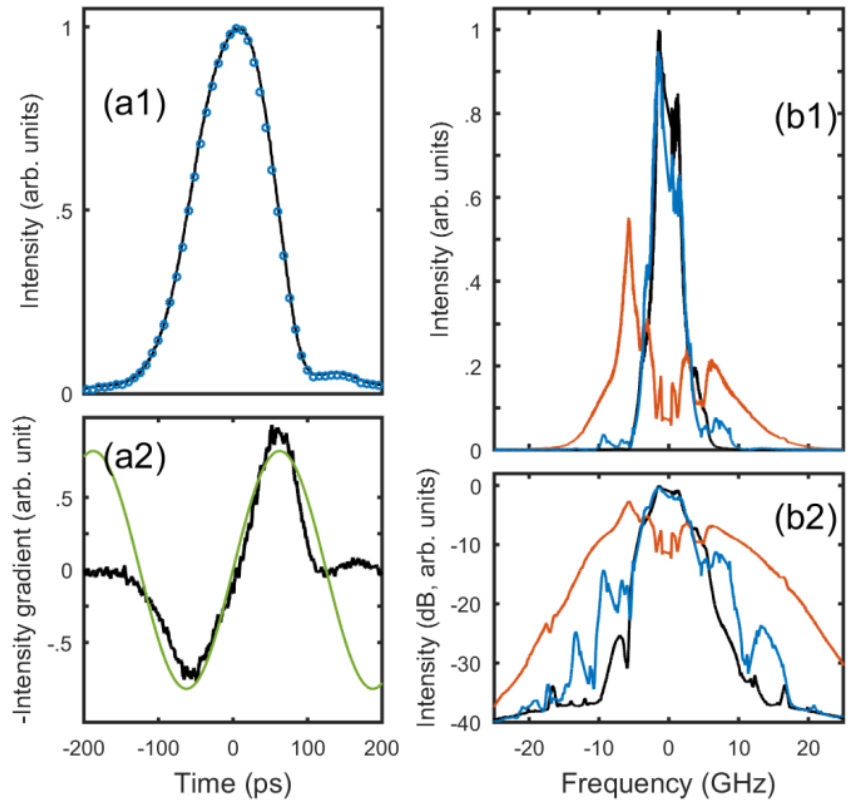

Fig. 4. Experimentally measured temporal (subplot (a1)) and spectral (subplot (b)) intensity profiles of the input pulse (black), without (red) and with (blue) phase modulation. Subplot (a2) shows the opposite of the temporal intensity gradient of the input pulse (black) compared with a sinusoidal fit (green). The accumulated $B$-integral is $B=5 \mathrm{rad}$.

Lastly, we have assessed the effectiveness of the proposed approach in the context of Raman amplification. The results obtained with on/off gain of $9 \mathrm{~dB}$, leading to a B-integral of $4.5 \mathrm{rad}$, are shown in Fig. 6. Once again, the external phase modulation enables preservation of the spectral intensity profile to a large extent, and yet the small side lobes present in the output spectrum can be ascribed to the input temporal intensity profile. 

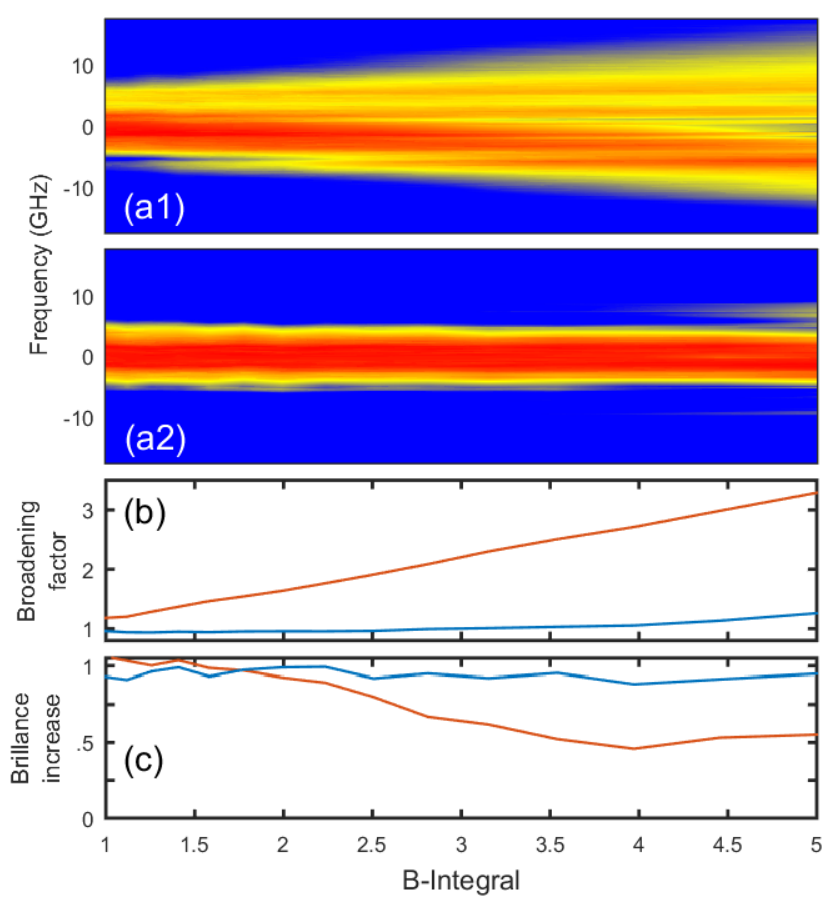

Fig. 5. (a) Experimentally measured evolution of the frequency spectrum (logarithmic scale, $17 \mathrm{~dB}$ dynamics) with the accumulated $B$ integral, without (subplot 1) and with (subplot 2) phase pre-correction. (b)-(c) Evolutions of the rms broadening factor and the brilliance increase of the pulse. The results obtained without and with phase correction are plotted with red and blue lines, respectively.

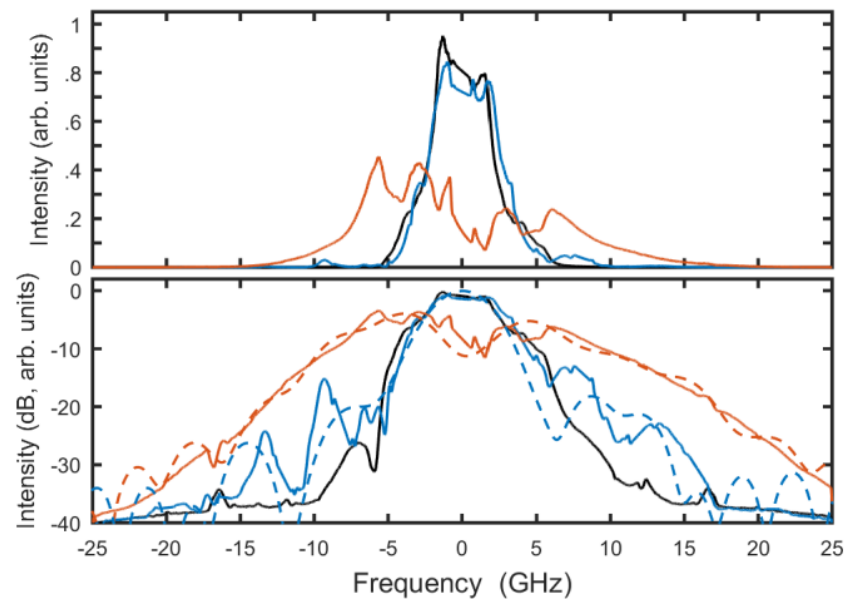

Fig. 6. Experimentally measured spectral intensity profiles at the entrance (black) and the exit of a Raman amplifier without (red) and with (blue) phase pre-compensation. Also shown are the results that can be numerically inferred assuming a close-to-Fourier-transform input waveform (dashed). The accumulated B-integral is B $=4.5 \mathrm{rad}$.

In conclusion, we have proposed and experimentally demonstrated a new, effective method to mitigate the impact of SPM on optical pulses of several tens of picoseconds duration propagating through a nonlinear medium. Imparting a sinusoidal phase modulation to the pulse in the time domain enables us to counteract the deleterious effects of SPM to a large degree. Proof-of-principle experiments have confirmed the success of the proposed scheme in cancelling high levels of distortion of the pulse spectrum. From the experimental standpoint, we were only constrained by the maximum amplitude of phase modulation that could be imparted by the modulator being used $(A=2.25 \mathrm{rad})$. But much stronger modulations are currently feasible $[1,14]$.

We believe that the method demonstrated here will prove useful for applications in the field of high-power pulse amplification, particularly with pulses of around a hundred picoseconds duration for which methods such as CPA are not practical due to the difficulty of adequately stretching them through a dispersive element. Temporal phase correction can, in this case, be accomplished prior to amplification. Our scheme can handle high pulse repetition rates. For pulse durations shorter than tens of picoseconds, it will be possible to combine the CPA method with our proposed technique into a hybrid scheme. In the absence of CPA, the interplay between GVD and SPM upon pulse propagation in a normally dispersive fiber may lead to more complex pulse dynamics resulting from the development of a close-to-linear frequency chirp [15-17]. In this case, the guidelines given in the present paper should be adjusted and the efficiency of the proposed approach will diminish, but noticeable correction of nonlinear impairments will be still possible. Ultimate performance of our phase-only pre-shaping method may be reached with an electrical arbitrary waveform generator combined with a feedback loop and learning or genetic algorithms.

Funding. Région Bourgogne (Pari Photcom) ; Agence Nationale de la Recherche (Labex Action ANR-11-LABX-01-01) ; Program FEDER-FSE Bourgogne 2014-2020.

Acknowledgment. The article has benefited from the PICASSO experimental platform of the University of Burgundy.

\section{References}

1. B. W. Plansinis, W. R. Donaldson, and G. P. Agrawal, J. Opt. Soc. Am. B 32, 15501554 (2015).

2. R. H. Stolen and C. Lin, Phys. Rev. A 17, 1448-1453 (1978).

3. G. P. Agrawal, Nonlinear Fiber Optics, Fourth Edition (Academic Press, San Francisco, CA, 2006).

4. D. J. Richardson, J. Nilsson, and W. A. Clarkson, J. Opt. Soc. Am. B 27, B63-B92 (2010).

5. D. Strickland and G. Mourou, Opt. Commun. 56, 219-221 (1985).

6. D. N. Schimpf, E. Seise, J. Limpert, and A. Tünnermann, Opt. Express 17, 49975007 (2009).

7. M. E. Fermann, V. I. Kruglov, B. C. Thomsen, J. M. Dudley, and J. D. Harvey, Phys. Rev. Lett. 84, 6010-6013 (2000)

8. L. Shah, Z. Liu, I. Hartl, G. Imeshev, G. C. Cho, and M. E. Fermann, Opt. Express 13, 4717-4722 (2005).

9. S. Zhou, L. Kuznetsova, A. Chong, and F. W. Wise, Opt. Express 13, 4869-4877 (2005).

10. S. Boscolo, L. K. Mouradian, and C. Finot, J. Opt. 18, 105504 (2016).

11. N.L.Markaryan, L.Kh.Muradyan and T.A.Papazyan, Sov. J. Quantum Electronics 21, 783-785 (1991).

12. M. Oberthaler and R. A. Höpfel, Appl. Phys. Lett. 63, 1017-1019 (1993).

13. K. Hammani, C. Finot, and A. Picozzi, Opt. Commun. 284, 2594-2603 (2011).

14. A. A. Godil, B. A. Auld, and D. M. Bloom, IEEE J. Quantum Electron. 30, 827-837 (1994).

15. D. Anderson, M. Desaix, M. Lisak, and M. L. Quiroga-Teixeiro, J. Opt. Soc. Am. B 9, 1358-1361 (1992).

16. C. Finot, L. Provost, P. Petropoulos, and D. J. Richardson, Opt. Express 15, 852 864 (2007).

17. C. Finot and S. Boscolo, J. Opt. Soc. Am. B 33, 760-767 (2016). 